\title{
Desarrollo de filtros interferenciales para emisores fotoluminiscentes basados en silicio poroso
}

\author{
V.TORRES', R.J.MARTÍN'1, S.MANOTAS², F.AGULLÓ2, J.M. MARTÍNEZ DUART ${ }^{1}$ \\ 'Departamento de Física Aplicada C-12,Universidad Autónoma de Madrid,Cantoblanco,Madrid. \\ ${ }^{2}$ Instituto de Ciencia de Materiales de Madrid,Consejo Superior de Investigaciones Científicas,Cantoblanco,Madrid.
}

\begin{abstract}
Las propiedades fotoluminiscentes y electroluminiscentes en el visible del silicio poroso hacen de éste un material muy interesante para el desarrollo de disposotivos optoelectrónicos. Para la obtención de dispositivos de calidad es necesario reducir la semianchura del espectro de luminiscencia del silicio poroso, típicamente de unos $100 \mathrm{~nm}$, para conseguir una emisión monocromática. Esto puede conseguirse formando sobre la capa luminiscente una estructura multicapa, también de silicio poroso, que actúe a modo de filtro interferencial. Así se consigue estrechar el rango de emisión cuanto se desee, sencillamente diseñando el filtro de manera conveniente. En este artículo se estudian las propiedades ópticas de las capas de silicio poroso para el posterior diseño de estos filtros.
\end{abstract}

Palabras clave: silicio poroso, multicapa, filtro interferencial, dispositivos optoelectrónicos, propiedades ópticas

\section{Development of interference filters for porous silicon based photoluminescent devices}

Porous silicon's photoluminescent and electroluminescent properties make it a very interesting material for the development of optoelectronic devices. To obtain high quality devices it is mandatory to narrowen the porous silicon luminescent spectrum, tipically about $100 \mathrm{~nm}$ wide. This can be obtained by forming a porous silicon multilayer structure on the luminescent layer that acts as an interference filter. Thus, the emission spectrum can be narrowed as much as wished by simply designing the filter in the appropriate way. In this article, the optical properties of porous silicon layers are studied for future filter design.

Keywords: porous silicon, multilayer, interference filter, optoelectronic devices, optical properties.

\section{INTRODUCCIÓN}

Desde que a principios de los años 90 se descubrieron las propiedades fotoluminiscentes [1] y electroluminiscentes [2] de alta eficiencia en el visible del silicio poroso (SP), el interés por este material aumentó de manera considerable. En condiciones normales, el espectro luminiscente tiene una forma aproximadamente gaussiana, con un máximo seleccionable en el rango visible y una anchura total a media altura del orden de $100 \mathrm{~nm}[3,4]$. La obtención de dispositivos optoelectrónicos de calidad plenamente integrados en silicio, que permitan prescindir de los compuestos III-V y II-VI, pasa por conseguir una luminiscencia monocromática por parte de las capas de silicio poroso. Así, en el presente trabajo se plantea la formación de estructuras multicapa de silicio poroso sobre capas luminiscentes, también de silicio poroso, de forma que puedan actuar a modo de filtro de cuarto de onda y estrechar así el espectro de emisión característico del SP (fig. 1). Las estructuras obtenidas han sido caracterizadas mediante medidas de espectrofotometría, microscopía electrónica de barrido (SEM) y microespectroscopía de fotoluminiscencia.

\section{PROCEDIMIENTO EXPERIMENTAL.}

Para la formación del silicio poroso partimos de obleas de silicio monocristalino tipo p, de orientación (100) y resistividad 0.1-0.5 $\Omega \cdot \mathrm{cm}$. En la parte posterior de la oblea se forma un contacto óhmico de aluminio para asegurar una buena conducción eléctrica. El silicio poroso se obtiene por ataque electroquímico en una disolución de HF (48\% vol.) y etanol (98\% vol.) en una proporción de 1:1. El proceso tiene lugar en una célula electroquímica de teflón bajo iluminación procedente de una lámpara halógena.
Para realizar los experimentos de caracterización óptica se han producido tres series de capas individuales de SP con distintas densidades de corriente aplicada: 25, 50 y $100 \mathrm{~mA} / \mathrm{cm}^{2}$. Cada una de estas series consta de cinco muestras, con tiempos de ataque electroquímico de 15, 30, 45, 60 y $90 \mathrm{~s}$. De este modo podemos determinar sistemáticamente el índice de refracción y el espesor de las capas obtenidas en función de la densidad de corriente aplicada, $J$, y del tiempo de anodización. Las medidas de reflectancia difusa se han llevado a cabo utilizando un espectrofotómetro Jasco V-560 de doble haz (apertura de rendija $2 \mathrm{~nm}$, resolución fotométrica $0.3 \%$ de $\mathrm{T}$ ) provisto de una esfera integradora de $6 \mathrm{~cm}$ de diámetro. Las imágenes SEM se han obtenido mediante un microscopio electrónico de barrido de la marca Philips, modelo XL30.

Para las medidas de fotoluminiscencia se han formado capas a 40 $\mathrm{mA} / \mathrm{cm}^{2}$ durante $180 \mathrm{~s}$ en una disolución HF/etanol 2:1. La longitud de onda de excitación utilizada es de $514.5 \mathrm{~nm}$ en un spot de 1 micra de diámetro. Los espectros de fotoluminiscencia han sido tomados con un espectrómetro Renishaw Ramascope 2000.

\section{RESULTADOS Y DISCUSIÓN.}

\subsection{Caracterización óptica de las capas individuales}

Para la determinación del índice de refracción de las capas individuales se han llevado a cabo medidas de reflectancia difusa en el rango 400-800 nm. Como era de esperar se observan franjas de interferencia en los espectros obtenidos (fig. 2). Calculando las diferencias de cami- 


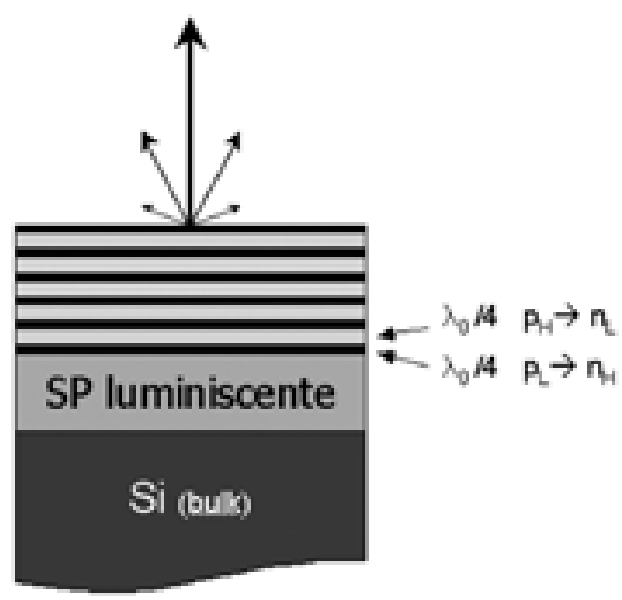

Fig. 1: La estructura multicapa actúa a modo de filtro interferencial, estrechando el espectro de emisión de la capa luminiscente de silicio poroso. Las capas de alta porosidad $\left(\mathrm{p}_{\mathrm{H}}\right)$ se corresponden con un índice de refracción bajo $\left(\mathrm{n}_{\mathrm{L}}\right)$, y viceversa.

no óptico puede demostrarse a partir de expresiones bien conocidas [5] que la posición de los máximos de interferencia viene dada por

$$
\lambda_{i}=\frac{2 d}{m_{i}} \sqrt{n\left(\lambda_{i}\right)^{2}-\sin ^{2} \theta}
$$

donde $d$ es el espesor de la capa, $n$ su índice de refracción, $\theta$ el ángulo de incidencia (en este caso $\theta=10^{\circ}$ ) y $m$ el orden de interferencia. Hemos comprobado que la expresión (1) se verifica en todos los casos. De esta forma, al aumentar el tiempo de ataque electroquímico de las capas de SP, se obtiene un mayor espesor que produce un mayor número de máximos y mínimos en un intervalo dado de longitudes de onda, como puede observarse en la fig. 2.

Se ha medido el espesor de las capas de manera directa mediante microscopía electrónica de barrido en sección transversal. En la fig. 3 puede observarse que el espesor de las capas es directamente proporcional al tiempo de anodización. Así, por ejemplo, se ha obtenido una velocidad de formación de $22 \mathrm{~nm} / \mathrm{s}$ para una densidad de corriente de $25 \mathrm{~mA} / \mathrm{cm}^{2}$, y de $73.5 \mathrm{~nm} / \mathrm{s}$ para $100 \mathrm{~mA} / \mathrm{cm}^{2}$.

Se ha modelizado el índice de refracción del silicio poroso mediante una mezcla homogénea de tres medios: aire (correspondiente a los poros), silicio cristalino y óxido de silicio. Es posible hacer esta estimación debido a que las estructuras del SP son un orden de magnitud menor que la longitud de onda de la luz [6]. Así, según el modelo propuesto, el índice de refracción del silicio poroso podrá expresarse mediante:

$$
\mathrm{n}_{\mathrm{SP}}(\lambda)=\mathrm{p} \cdot \mathrm{n}_{\text {aire }}(\lambda)+(1-\mathrm{p})\left[\mathrm{f} \cdot \mathrm{n}_{\mathrm{Si}}(\lambda)+(1-\mathrm{f}) \cdot \mathrm{n}_{\mathrm{SiO} 2}(\lambda)\right]
$$

donde $p$ es la fracción de aire (porosidad) y $f$ la fracción de silicio cristalino que no se ha oxidado. Los valores del índice de refracción del silicio cristalino y del óxido de silicio se encuentran tabulados en la literatura [7]. Con estas expresiones, y conocido el espesor de las capas que previamente ha sido obtenido mediante SEM, podemos ajustar, para cada serie de muestras, el valor de p y f, y obtener así el índice de refracción efectivo correspondiente a la capa de silicio poroso. Los resultados obtenidos vienen resumidos en la fig. 4 . En todos los casos la relación de dispersión es de tipo Cauchy y puede observarse cómo el índice de refracción de las capas disminuye con la densidad de corriente de formación de las capas porosas. Este comportamiento puede ser asociado al hecho de que cuanto mayor es la porosidad de las

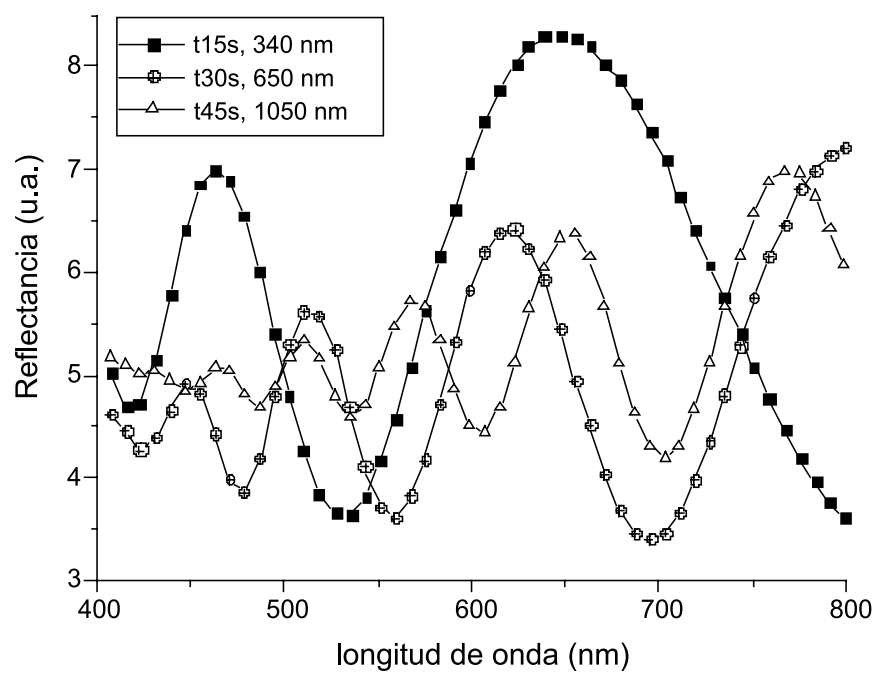

Fig 2: Espectros de reflectancia de tres muestras de la serie de 25 $\mathrm{mA} / \mathrm{cm}^{2}$

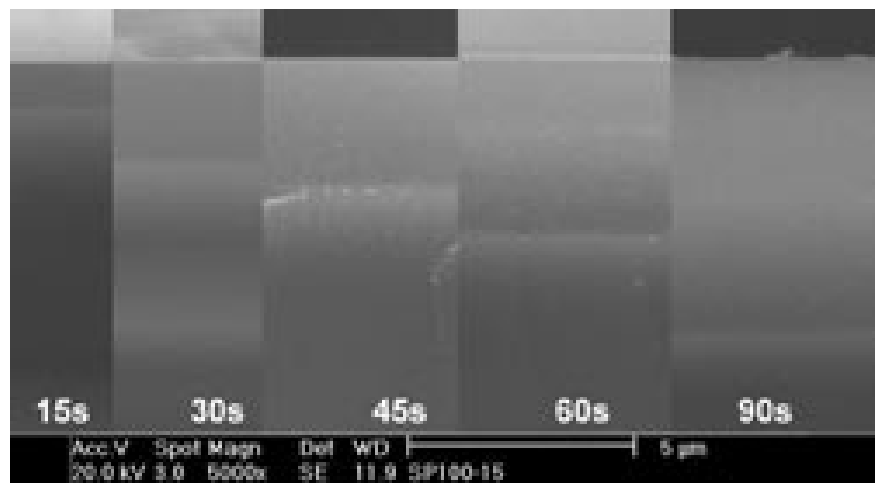

Fig. 3: Imágenes de SEM combinadas de la serie de muestras de 100 $\mathrm{mA} / \mathrm{cm}^{2}$

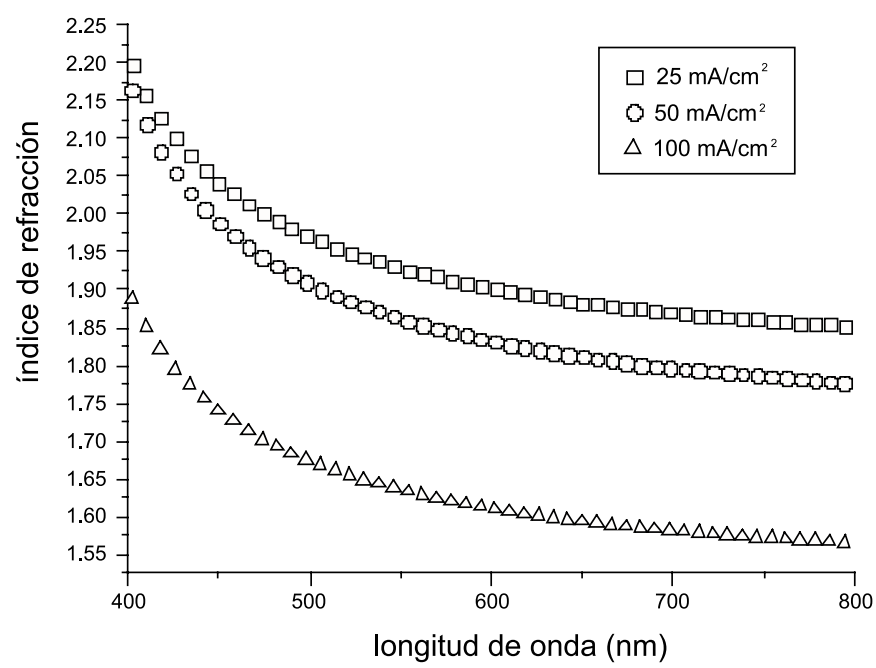

Fig 4: Índice de refracción de las capas de silicio poroso, según la densidad de corriente aplicada durante la anodización.

muestras mayor es la fracción de aire que contienen, produciéndose una reducción del índice de refracción efectivo. Pero al mismo tiempo, esta disminución puede ser asociada a una mayor fracción de óxido de silicio presente en las muestras, debido a un aumento de la superficie específica al aumentar la porosidad. 


\subsection{Capas fotoluminiscentes}

Por otra parte, se han llevado a cabo medidas de fotoluminiscencia en sección transversal en capas de silicio poroso con espesores típicos de 4 micras. La fig. 5 muestra un espectro típico de fotoluminiscencia en profundidad de las capas de silicio poroso. Puede observarse que el máximo de luminiscencia se mantiene constante alrededor de $1.7 \mathrm{eV}$ en todo el grosor de la muestra. Los resultados nos indican que la homogeneidad de las capas obtenidas es alto, ya que el pico de máxima luminiscencia depende en gran medida del tamaño de los nanocristales que componen el silicio poroso, así como de otros factores como la porosidad [8]. Por tanto, el método utilizado para la fabricación de las capas de silicio poroso proporciona una alta homogeneidad en espesor, lo que repercute positivamente en el comportamiento de dispositivos fabricados con este material.

\subsection{Multicapas}

Finalmente, y una vez establecida una relación empírica entre el índice de refracción y la densidad de corriente de formación de las capas de silicio poroso, se han fabricado estructuras multicapa. Para obtener estas estructuras se varía periódicamente la densidad de corriente aplicada [9] con lo que se consigue un perfil variable de porosidad, tal y como puede observarse en la fig. 6 . La porosidad en función de la profundidad da lugar a un perfil del índice de refracción en profundidad, controlado y de interfases abruptas. Se han determinado las propiedades ópticas de estas estructuras en el intervalo visible de longitudes de onda (fig. 7). Puede observarse cómo se obtiene un espectro de reflectancia en los que se producen fenómenos interferenciales. Los extremos de interferencia coinciden con los esperados, tal y como podemos comprobar a partir del espectro simulado que se muestra en la fig. 7 . Sin embargo, debido al gran espesor de las estructuras formadas, los fenómenos de absorción y dispersión en el silicio poroso provocan una pérdida parcial de fase que hace que los espectros experimentales se desvíen de los resultados esperados. Este fenómeno es mayor a longitudes de onda menores, como se refleja en la figura 7. Por tanto es necesario reducir el espesor total de las estructuras multicapa para minimizar este efecto. Al mismo tiempo sería interesante reducir la absorción por parte de las capas individuales de silicio poroso. Esto puede conseguirse realizando, entre otros, procesos de oxidación de las capas porosas.

\section{CONCLUSIONES.}

Se ha demostrado la posibilidad de obtener capas homogéneas de silicio poroso de índice de refracción controlado de una manera relativamente sencilla mediante el ataque electroquímico de silicio monocristalino. Del mismo modo también se han obtenido estructuras multicapa de interfases abruptas, lo que ha dado lugar a un perfil controlado de índices de refracción en profundidad. Por tanto, combinando las propiedades fotoluminiscentes del silicio poroso con estructuras multicapa basadas en ese mismo material es posible desarrollar dispositivos optoelectrónicos foto o electroluminiscentes integrados en silicio. Mediante variaciones en las propiedades de las capas luminiscentes y de las estructuras multicapa, se está en condiciones de poder filtrar la emisión del silicio poroso, reduciéndola a un estrecho intervalo de longitudes de onda.

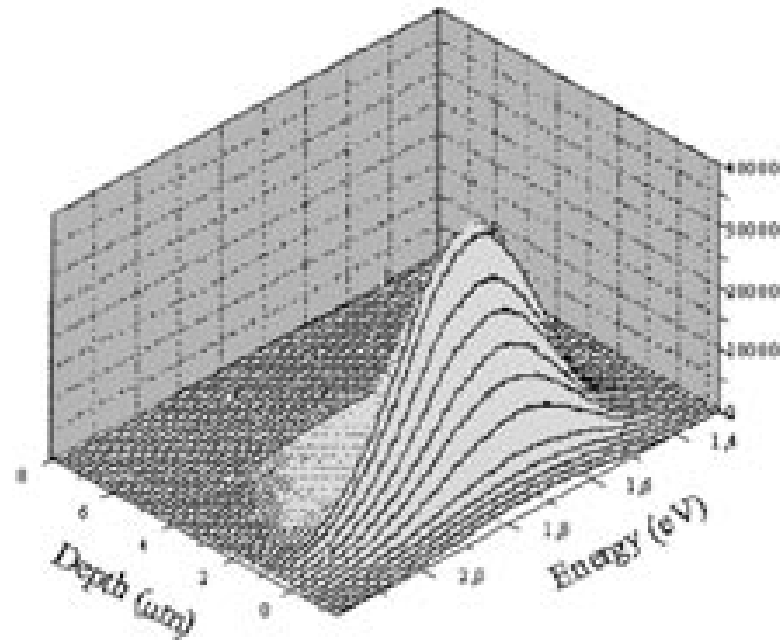

Fig. 5: Espectro de fotoluminiscencia en profundidad de una capa de silicio poroso de 4 micras de grosor.

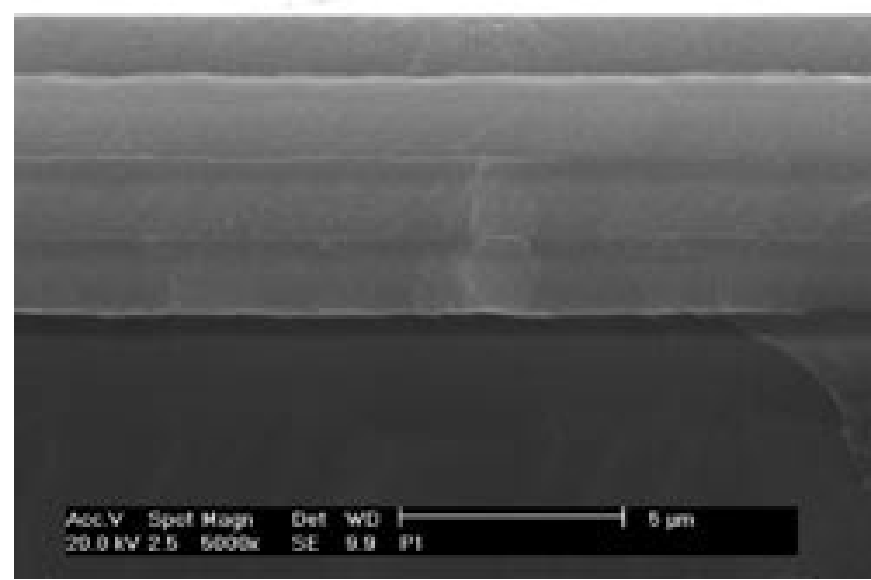

Fig. 6: Imagen de microscopía electrónica de barrido de una estructura de 4 pares de capas, con un espesor total de unas 6.5 micras

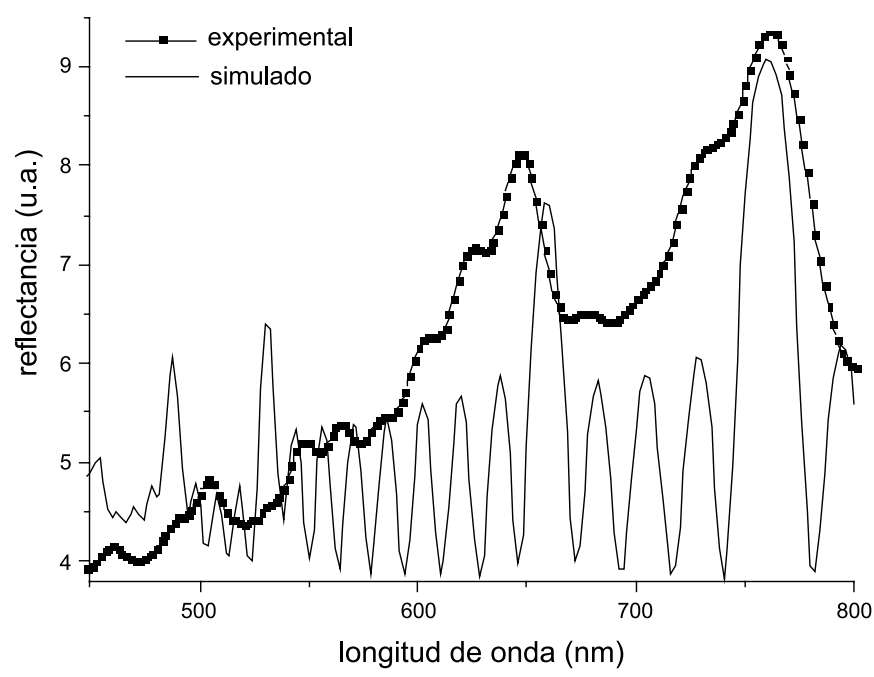

Fig. 7: Espectro de reflectancia de una estructura compuesta por ocho capas. Se observan los fenómenos de interferencia sobre un fondo producido por la pérdida parcial de fase. 


\section{AGRADECIMIENTOS.}

Los autores quisieran expresar su agradecimientoa D. Graciano Hueso por su importante contribución técnica al desarrollo del presente trabajo. Este trabajo ha sido financiado por el Ministerio de Ciencia y Tecnología a través de la Comisión Interministerial de Ciencia y Tecnología, mediante el Proyecto de Investigación MAT2000-0375C02-01.

\section{BIBLIOGRAFÍA}

1. L. T. Canham, "Silicon quantum wire array fabrication by electrochemical and chemical dissolution of wafers" Appl. Phys. Lett. 57, 1046 (1990)

2. N. Koshida and H. Koyama, "Visible electroluminescence from porous silicon" Appl. Phys. Lett. 60, 347 (1992)
3. R. T. Collins, P. M. Fauchett and M. A. Tischler, "Porous silicon: from luminescence to leds" Phys. Today, January 1997, p. 24

4. O. Bisi, S. Ossicini and L. Pavesi, "Porous silicon: a quantum sponge structure for silicon based optoelectronics" Surf. Sci. Rep. 38(1-3), 5 (2000)

5. O. S. Heavens, "Optical properties of thin solid films", Dover Publications, New York (1965)

6. R. J. MartÃn-Palma, L. Pascual, P. Herrero and J. M. MartÃnez-Duart, "Direct determination of grain sizes, lattice parameters, and mismatch of porous silicon" Appl. Phys. Lett. 81(1), 25 (2002)

7. E. D. Palik (ed.), “Handbook of optical constants of solids", Academic Press Inc. Washington DC (1985)

8. A. G. Cullis, L. T. Canham, P. D. J. Calcott, "The structural and luminescence properties of porous silicon" J. Appl. Phys. 82(3), 909 (1997)

9. M. G. Berger, M. Thanissen, R. Aren-Fischer, H. Mander, H. Lath, M. Arntzen, W. Theiss, "Investigation and design of optical-properties of porosity superlattices" Thin Solid Films, 255, 313 (1995)

Recibido: 1.2.03

Aceptado: 30.11 .03 